\title{
INFLUÊNCIA DO BIOFEEDBACK EM PACIENTES COM SEQUELA DE ACIDENTE VASCULAR CEREBRAL
}

INFLUENCE OF BIOFEEDBACK IN PATIENTS WITH SEQUELAE OF STROKE

\section{Tatiana dos Anjos Pimentel ${ }^{a}$, Bruna Carla Pereira da Silva ${ }^{b}$, Cláudia Kelly Vieira Silva ${ }^{c}$, Juliana de Oliveira Souza ${ }^{\mathrm{d}}$, Priscila de Oliveira Januário ${ }^{\mathrm{e}}$, Ariela Torres Cruz $^{\mathrm{f}}$}

\author{
atati-anjos@hotmail.com, bbpfisio2016@gmail.com, ckellyvieirabm@hotmail.com, djufisio_souza@yahoo.com.br, epri.januario@gmail.com, \\ fariela_tcruz@yahoo.com.br \\ Centro Universitário de Barra Mansa - Barra Mansa (RJ), Brasil
}

Data de recebimento de artigo: 22/01/2017

Data de aceite do artigo: 02/06/2017

\section{RESUMO}

Introduçáo: $\mathrm{O}$ acidente vascular cerebral (AVC) é considerado o principal comprometimento neurológico que causa incapacidade funcional em adultos. Várias técnicas de reabilitaçáo, como o treinamento de Biofeedback, vêm sendo inseridas às modalidades terapêuticas para estimular as funçóes motoras desses pacientes. Objetivos: Avaliar o efeito do Biofeedback em pacientes com sequela de AVC. Materiais e métodos: Estudo clínico randomizado, duplo-cego com amostra de 16 indivíduos divididos em dois grupos: experimental (GE) e controle (GC). Os participantes do GE foram submetidos a 16 atendimentos, 2 vezes por semana, utilizando-se o Biofeedback e avaliados através da goniometria para mensurar a amplitude de movimento (ADM) de flexão e extensão de punho antes, depois e um mês após o término do tratamento. Os participantes do GC foram avaliados nos mesmos momentos. Resultados: Verificouse que no GE houve um aumento da ADM de extensão de punho logo depois ( $\mathrm{p}=0,0151$ ) e um mês após o término do tratamento $(\mathrm{p}=0,0227)$, sendo que não houve resultado estatisticamente significativo para a análise do movimento de flexão do GE e GC. Ao comparar os grupos, observou-se que não houve diferença entre eles. Conclusóes: $\mathrm{O}$ tratamento proposto se mostrou eficaz para o aumento da ADM de extensão de punho dos participantes do GE, porém, estatisticamente não houve diferença entre os grupos analisados.

Palavras-chave: Acidente vascular cerebral; biofeedback; espasticidade muscular; paresia.

\section{ABSTRACT}

Introduction: Stroke is considered the main neurological impairment that causes functional disability in adults. Several rehabilitation techniques, such as Biofeedback therapy, have been inserted to therapeutic modalities to stimulate the motor functions of these patients. Objectives: To evaluate the effect of Biofeedback on patients with sequelae of stroke. Materials and methods: Clinical, randomized, double-blind study on 16 individuals divided into two groups: experimental (EG) and control (CG). EG participants underwent 16 medical services, two times a week, using Biofeedback and were assessed by goniometry to measure range of motion (ROM) of wrist flexion and extension before, after and one month after the end of the treatment. CG participants were assessed at the same time. Results: We found that in EG there was an increase of ROM of wrist extension right after $(\mathrm{p}=0.0151)$ and one month after the end of the treatment $(\mathrm{p}=0.0227)$, and there was no statistically significant results for the analysis of flexion motion of EG and CG. When comparing groups, we observed no difference between them. Conclusions: The proposed treatment proved to be effective for increasing the ROM of wrist extension of EG participants; however, there was no statistical difference between the groups analyzed.

Keywords: Stroke; biofeedback; muscle spasticity; paresis. 


\section{Introdução}

$\mathrm{O}$ acidente vascular cerebral (AVC), de acordo com a Organização Mundial da Saúde (OMS), é definido como um sinal clínico de rápido desenvolvimento de perturbação focal da função cerebral, supostamente de origem vascular e com mais de 24 horas de duração ${ }^{1}$. No Brasil, o AVC se destaca no topo do ranking de mortalidade, representando a principal causa de óbitos dentre todas as doenças e sendo também considerado uma das injúrias que mais ameaça a qualidade de vida².

Existem dois tipos de AVC, o isquêmico e o hemorrágico. O primeiro é o mais comum, afetando cerca de $80 \%$ dos indivíduos acometidos, e acontece por obstrução decorrente de um coágulo que bloqueia ou impede o fluxo sanguíneo, privando o cérebro de oxigênio e dos nutrientes essenciais. O AVC hemorrágico, por sua vez, ocorre quando os vasos se rompem, ocasionando derramamento de sangue no interior ou ao redor do cérebro ${ }^{3}$.

Estima-se que cerca de $40 \%$ a $50 \%$ dos indivíduos que sofrem AVC morrem após seis meses. A maioria dos indivíduos exibirá deficiências neurológicas e incapacidades residuais significativas, o que faz desta patologia a primeira causa de incapacitação funcional no mundo ocidental $^{4}$. Existem variaçóes na gravidade das sequelas apresentadas após o AVC, o que irá predizer o comprometimento e a dependência funcional para realização de suas atividades cotidianas, como alimentar-se, banhar-se, vestir-se, deambular, deitar-se e levantar-se ${ }^{5}$. Dentre as diversas sequelas, destacam-se a espasticidade e a hemiparesia ${ }^{3}$.

A espasticidade, definida como resistência do músculo ao alongamento, é dependente da velocidade e se baseia na ativação dos reflexos tônicos em resposta ao estiramento, sendo mais acentuada nos músculos que se opóem à ação da gravidade 6 . Já a hemiparesia é caracterizada pela diminuição dos movimentos voluntários em um hemicorpo, apresentando alteraçóes musculares, sensitivas e cognitivas 5 .

O objetivo do tratamento após o AVC geralmente está relacionado a um retreinamento da atividade voluntária dos extensores de punho, dos abdutores do braço e dos dorsiflexores do tornozelo, no intuito de aumentar a resposta muscular ${ }^{7}$.

Nos últimos anos, vários recursos fisioterápicos vêm sendo sugeridos para amenizar as sequelas ocasionadas pelo AVC, através de técnicas como mobilização, alongamento, ortostatismo, eletroestimulação, crioterapia e outras ${ }^{8}$. Dentre as diversas técnicas destaca-se o Biofeedback Eletromiográfico (EMG) que, desde 1960 , tem sido utilizado no tratamento da hemiparesia. Numerosos estudos têm demonstrado a eficácia dessa técnica; a maioria deles relata que o Biofeedback EMG é capaz de conquistar melhorias, mesmo no estado crônico, no tratamento da hemiparesia superior' ${ }^{9}$.

Resultados positivos da atividade motora voluntária com a utilização do biofeedback baseiam-se, supostamente, no mecanismo de plasticidade do sistema nervoso central (SNC), fato que ocorre a partir da reorganização da função neural resultante da adaptação de uma nova demanda. $O$ biofeedback permite realizar treinamento de facilitação, inibição e coordenação motora de maneira seletiva ${ }^{10}$.

Várias técnicas de reabilitação como o treinamento de Biofeedback, que servem para estimular as funçóes motoras, tornaram-se objeto de estudos e vêm sendo inseridas às modalidades terapêuticas. Essa técnica permite que o paciente tenha um feedback imediato ao exercício, possibilitando a ele monitorar a melhora de sua atividade motora e cognitiva, ainda que esta não seja visível de imediato, servindo como estímulo à continuação do tratamento ${ }^{11}$.

Dessa forma, este estudo teve como objetivo avaliar o efeito do Biofeedback EMG na extremidade distal do membro superior parético de pacientes com sequela de AVC.

\section{Metodologia}

Trata-se de um estudo experimental clínico randomizado e duplo-cego, que teve início após a aprovação pelo Comitê de Ética em Pesquisa do Centro Universitário de Barra Mansa (UBM) sob protocolo número 970.316. Participaram do estudo 16 pacientes, dos dois sexos, com idade entre 37 e 73 anos, em fase crônica de AVC e com hemiparesia espástica de membro superior, que foram escolhidos aleatoriamente no Centro Integrado de Saúde (CIS) de um centro universitário situado no interior do estado do Rio de Janeiro e que aceitaram fazer parte do estudo conforme o Termo de Consentimento Livre e Esclarecido (TCLE). Os participantes foram divididos em dois grupos iguais após sorteio aleatório: grupo experimental (GE) e grupo controle (GC); e continuaram com o tratamento fisioterápico convencional já realizado no CIS durante toda a pesquisa. Foram incluídos neste estudo pacientes em fase crônica de AVC (no mínimo 3 meses após a lesão), com no máximo grau 3 e mínimo grau +1 de espasticidade - de acordo com a escala de Ashworth Modificada -, indicação médica para fisioterapia, uma adequada compreensão e competências de comunicação, que apresentaram movimentos voluntários do membro superior parético, e que aceitaram fazer parte do estudo de acordo com o TCLE. Foram excluídos pacientes portadores de qualquer qualidade de demência, que apresentaram déficit auditivo 
e visual importante, afasia sensorial, fraturas em membros superiores, pacientes com sequela motora bilateral, e com grau de espasticidade superior a 3 ou inferior a 1 , de acordo com a Escala de Ashworth Modificada.

Os pacientes foram submetidos a uma avaliação (com um avaliador independente) antes do tratamento, na qual foram utilizados a escala de Ashworth Modificada e um goniômetro. Os pacientes do GE e do GC foram avaliados novamente com o goniômetro ao final do tratamento e um mês após seu término. Os 16 pacientes foram distribuídos em dois grupos após sorteio aleatório, sendo 8 pacientes no GE e 8 no GC. Após a avaliação inicial, os pacientes do GE foram submetidos a 16 atendimentos individuais utilizando o Biofeedback, duas vezes por semana, em um período de dois meses. Em um ambiente reservado, o paciente foi adequadamente sentado em uma cadeira, com os antebraços apoiados em uma mesa e em seguida foi realizada a higienização do local de fixação dos eletrodos com algodáo e álcool. Utilizou-se o aparelho Biofeedback EMG com eletrodos de cloreto de prata medindo $1 \mathrm{x} 1$ $\mathrm{cm}$, que foram posicionados no ventre dos músculos extensores de punho e dedos bilateralmente e fixados com fita adesiva, com os parâmetros no modo manual, zoom entre 0 e 9 , alarme acionando o alvo atingido na funçáo target (alerta quando a atividade do EMG está a $15 \%$ do alvo) e volume do sinal auditivo entre 0 e 9 com som dinâmico (o volume do som é aumentado de acordo com a aproximação da atividade EMG do alvo). Os pacientes foram orientados a olhar diretamente para o visor do aparelho durante o tratamento e a realizar a contração muscular máxima através do movimento de extensẫo de punho e dedos para determinar o alvo ao qual o paciente deveria chegar quando realizasse os exercícios. Posteriormente, os pacientes foram orientados a realizar contraçôes musculares isotônicas de 10 repetiçôes durante 10 minutos, com intervalo das contrações de 10 segundos, com o objetivo de tentar igualar a atividade do músculo do lado afetado com o lado sadio.

Os dados foram coletados e exportados para um sistema de banco de dados e analisados com auxílio do programa BioEstat, versão 5.0. Para verificar se eles seguiam uma distribuição normal, realizou-se o teste de normalidade de Shapiro-Wilk. Portanto, foi aplicado o teste paramétrico " $t$ " de Student para observações pareadas (dependentes) e não pareadas (independentes) com nível de significância de $\mathrm{p} \leq 0,05$.

\section{Resultados}

Os dados referentes às características dos participantes do estudo encontram-se na Tabela 1.
Tabela 1: Características gerais da amostra em relação a faixa etária, gênero, tempo de lesão e lado acometido.

\begin{tabular}{lcc} 
Variáveis & $\begin{array}{c}\text { Grupo } \\
\text { Experimental (n=8) } \\
\mathbf{N}(\%)\end{array}$ & $\begin{array}{c}\text { Grupo } \\
\text { Controle (n=8) } \\
\mathbf{N}(\%)\end{array}$ \\
Faixa etária & & \\
30 a 40 anos & $1(12,5)$ & $0(0)$ \\
41 a 50 anos & $0(0)$ & $0(0)$ \\
51 a 60 anos & $2(25)$ & $2(25)$ \\
Acima de 60 anos & $5(62,5)$ & $6(75)$ \\
Gênero & & \\
Feminino & $4(50)$ & $0(0)$ \\
Masculino & $4(50)$ & $8(100)$ \\
Tempo de lesão & & $6(75)$ \\
02 a 05 anos & $2(25)$ & $1(12,5)$ \\
06 a 10 anos & $2(25)$ & $1(12,5)$ \\
Acima de 10 anos & $4(50)$ & \\
Hemiparesia & & $5(62)$ \\
Direita & $2(25)$ & $3(38)$ \\
Esquerda & $6(75)$ & \\
\hline
\end{tabular}

Após o tratamento com Biofeedback EMG, houve um aumento significativo da amplitude de movimento de extensáo de punho no GE $(\mathrm{p}=0,0151)$, com manutenção um mês após o término do tratamento $(\mathrm{p}=0,0227)$. Ao comparar os grupos observou-se que não houve diferença estatística entre eles nos tempos antes e depois do tratamento $(\mathrm{p}=0,1230)$, assim como nos tempos depois e 1 mês após o término do tratamento $(\mathrm{p}=0,0839)$ (Gráfico 1).

Gráfico 1: Comparação da amplitude de movimento de extensão entre GE e GC antes, depois e 1 mês após o término do tratamento.

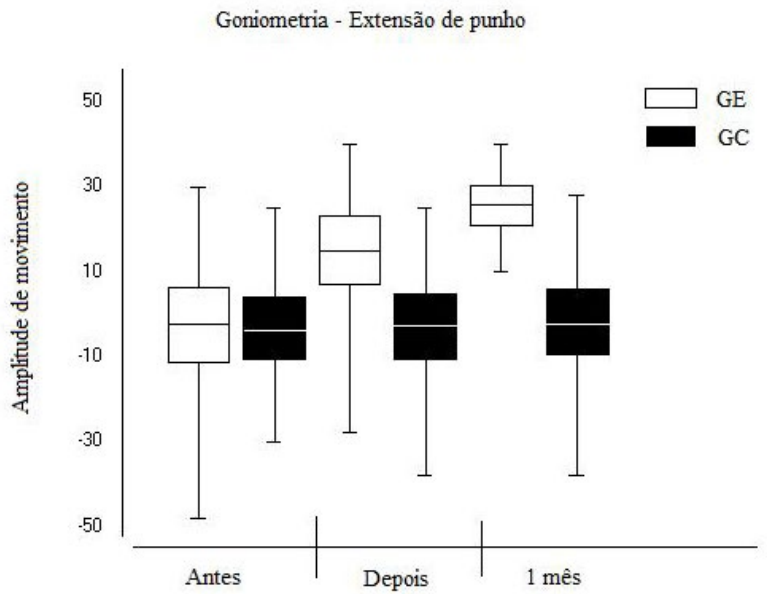

Com relação à amplitude de movimento de flexão de punho, verificou-se que não houve aumento da 
amplitude de movimento (ADM) do GE após o tratamento $(\mathrm{p}=0,1540)$, assim como no $\mathrm{GC}(\mathrm{p}=0,0646)$. Ao comparar os grupos, não foi possível verificar diferença estatística entre os resultados antes e depois do tratamento ( $\mathrm{p}=0,4583)$, assim como o de depois e 1 mês após o término do tratamento $(\mathrm{p}=0,2881)$ (Gráfico 2).

Gráfico 2: Comparação da amplitude de movimento de flexão entre GE e GC antes, depois e 1 mês após o término do tratamento.

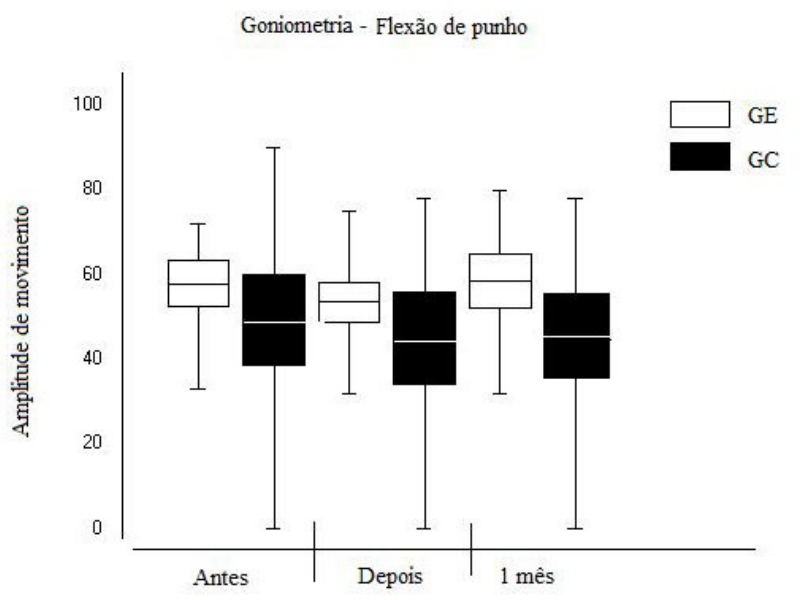

\section{Discussão}

Os artigos de pesquisa analisados em uma revisão sobre o emprego do Biofeedback no tratamento de doenças crônicas mostraram que os participantes eram dos dois sexos, com grande variabilidade de idade, desde crianças, adolescentes, adultos e idosos. Isso indica que o Biofeedback é uma ferramenta que pode ser utilizada numa ampla gama de indivíduos ${ }^{12}$.

Em relação à reabilitação de sujeitos que sofreram AVC, a maior parte da recuperação neurológica acontece dos 3 aos 6 primeiros meses após o evento, e estima-se que somente $5 \%$ deles continuem experimentando essa recuperaçáa ${ }^{13}$. Como neste estudo o menor tempo de lesáo apresentado pelos participantes foi de 2 anos, pode-se afirmar que possivelmente esses sujeitos encontravam-se com reduzida possibilidade de recuperaçáo espontânea.

Um estudo realizado para avaliar hemiparéticos crônicos submetidos à fisioterapia no formato de circuito de treinamento mostrou que esses pacientes têm possibilidade de melhorar sua mobilidade e autonomia funcional ${ }^{14}$, corroborando os dados apresentados por esta pesquisa, que mostram que mesmo em pacientes crônicos foi possível observar um ganho na ADM de extensão de punho.
Os benefícios obtidos podem estar associados à plasticidade neural. A literatura mostra que o nosso cérebro é mutável a cada nova experiência. Sendo assim, praticar atividades diversificadas, repetitivas e desafiadoras pode ser uma boa maneira de estimular o cérebro, provocando alteraçóes no padráo de funcionamento e de conexão das células cerebrais ${ }^{15}$. Acredita-se que os resultados positivos na amplitude de movimento de extensão ativa de punho dos participantes deste estudo derivam-se do treinamento repetitivo desse movimento, com estímulo visual e auditivo realizado durante o tratamento com Biofeedback EMG.

Um estudo, realizado com o objetivo de avaliar a eficácia do Biofeedback EMG como ferramenta para ativar o glúteo médio e eretores da espinha de pacientes com AVC em piscina terapêutica, mostrou que o uso isolado desse recurso foi capaz de melhorar o alinhamento do quadril e do tronco dos participantes, confirmando os resultados obtidos nesta pesquisa - na qual foi possível observar o aumento da ADM de extensão de punho apenas com o tratamento isolado do Biofeedback EMG ${ }^{16}$.

Em um estudo realizado com pacientes de paralisia facial periférica, observou-se um aumento da funçáo física e da atividade muscular após o tratamento com Biofeedback EMG e a manutenção desses resultados no período de um mês após o término do tratamento ${ }^{17}$. Esses dados reiteram nossos resultados, mostrando-se, apesar dos tratamentos serem em áreas e patologias distintas, de acordo com os princípios do Biofeedback EMG de reeducação motora, visto que neste estudo houve um aumento significativo da amplitude de movimento de extensão de punho sem reduçáo um mês após o término do tratamento.

Outras pesquisas mostraram o efeito positivo do biofeedback em situaçóes diversas, como redução do estresse e ansiedade em atletas ${ }^{18}$, melhora da incontinência urinária ${ }^{19,20}$, direcionamento na ativação muscular de pacientes com AVC em piscina terapêutica ${ }^{16}$ e melhora da marcha em pacientes com AVC e doença de Parkinson ${ }^{21}$. Apesar dos resultados positivos serem semelhantes a este estudo, as metodologias aplicadas diferem-se entre si.

O padrão flexor é predominante em membros superiores das vítimas de $\mathrm{AVC}^{22}$. A não alteração da $\mathrm{ADM}$ de flexáo de punho do GE pode ser associada ao fato de que somente o movimento de extensáo de punho e dedos foi solicitado durante o tratamento proposto, náo havendo assim nenhum estímulo aos flexores.

A dificuldade para recuperar a função do membro superior parético pode acontecer a partir de um fenômeno conhecido como "desuso aprendido", que se trata de um evento bem descrito na literatura e comprovado na 
experiência clínica predominantemente em pacientes hemiparéticos crônicos ${ }^{23}$. É possível atrelar essas afirmaçóes aos resultados deste estudo, no qual não foi observado aumento de ADM de punho no GC, a partir da perspectiva de negligência dos pacientes com o membro parético. Uma vez que não foi realizada atividade específica com a finalidade de ganho de ADM de punho, entende-se que os pacientes continuaram a náo utilizar o membro afetado.

Armagan, Tascioglu e Oner ${ }^{9}$ realizaram um estudo randomizado com 27 pacientes, contendo um grupo de tratamento com biofeedback e um grupo placebo. Com os resultados obtidos, observaram aumento da $\mathrm{ADM}$ de extensão de punho em ambos os grupos, todavia, ao compará-los, não foi observada diferença estatisticamente significativa. Neste estudo, apesar de náo ter sido possível verificar diferença entre os resultados do GE e do GC, constatou-se que o GE obteve aumento da ADM de extensão de punho, o que não ocorreu com o GC. Nos dois estudos, a diferença de resultados entre os grupos não tratados com biofeedback pode ser atribuída ao fato de que o grupo placebo treinava os movimentos da mesma forma que o grupo tratamento, porém sem o feedback auditivo e visual do aparelho - e neste estudo o GC náo realizava nenhuma tarefa específica que justificasse tal ganho.

O Biofeedback EMG é largamente utilizado como uma terapia independente, porém maiores resultados podem ser conquistados quando associada a outro recurso terapêutico, como foi mostrado em uma revisão de literatura relacionada ao tratamento fisioterapêutico nas disfunções sexuais em mulheres após tratamento de câncer ginecológico e de câncer de mama ${ }^{24}$. Visto isso, considera-se relevante a realização de estudos que aliem o tratamento do Biofeedback EMG com outras técnicas fisioterapêuticas específicas para investigar prováveis melhorias, complementando assim os achados deste estudo.

A maioria dos pacientes que sofrem um AVC terá sequelas, tornando a intervenção precoce do fisioterapeuta indispensável para a recuperação de movimentos perdidos/alterados, a fim de possibilitar uma melhor qualidade de vida e proporcionar uma independência cada vez mais significativa em suas atividades diárias ${ }^{25}$. Considera-se possível que resultados não favoráveis quanto ao ganho de ADM de flexão de punho resultem do fato de que nenhum estímulo de flexão de punhos e dedos foi dado aos pacientes do GE e do GC durante o tratamento, sendo trabalhado somente o movimento de extensão de punhos e dedos do GE.

\section{Conclusão}

Após análise dos resultados, foi possível concluir que o tratamento proposto contribuiu para o aumento da
ADM de extensão de punho no GE, porém, ao comparar os dados com os do GC, náo houve diferença estatística entre os grupos.

O Biofeedback EMG parece ser uma valiosa ferramenta para o tratamento das alteraçóes de pacientes com hemiparesia espástica, mas ainda são poucos os trabalhos publicados com embasamento científico que comprovem a eficácia do tratamento e sua repercussão na extremidade distal dos membros superiores de pacientes hemiparéticos por sequela de AVC.

Sugere-se que sejam realizados outros estudos que discorram da mesma temática, com um número maior de participantes, um maior tempo de tratamento e que associem outras técnicas, possibilitando avaliar mais extensamente os efeitos desta abordagem.

\section{Referências}

1. Correia ACZ, Silva JDS, Silva LVC, Oliveira DA, Cabral ED. Crioterapia e cinesioterapia no membro superior espástico no acidente vascular cerebral. Fisioter Mov. 2010;23(4):555-63.

2. Resende JSF, Brito JI, Sá ACAM. Medo de quedas em pacientes hemiparéticos pós-acidente vascular cerebral e o potencial para o risco de quedas [monografia]. Goiânia: Centro de Estudos Avançados e Formação Integrada; 2010.

3. O'Sullivan SB. Fisioterapia: avaliação e tratamento. $5^{\mathrm{a}}$ ed. Sáo Paulo: Manole; 2010. 1506 p.

4. Teixeira CP, Silva LD. As incapacidades físicas de pacientes com acidente vascular cerebral: açóes de enfermagem. Enferm glob. 2009;15:1-12.

5. Benvegnu AB, Gomes LA, Souza CT, Cuadros TBB, Pavão LW, Ávila SN. Avaliação da medida de independência funcional de indivíduos com sequelas de acidente vascular encefálico (AVE). Ciência\&Saúde. 2008;1(2):71-7.

6. Hüter-Becker A, Dölken M. Fisioterapia em neurologia. $1^{\text {a }}$ ed. São Paulo: Santos; 2008. 409 p.

7. Robertson V, Ward A, Low J, Reed A. Eletroterapia explicada: princípios e prática. $4^{\mathrm{a}} \mathrm{ed}$. Rio de Janeiro: Elsevier; 2011.520 p.

8. FeliceTD,SantanaLR. Recursosfisioterapêuticos (crioterapiae termoterapia) na espasticidade: revisão de literatura. Rev Neurocienc. 2009;17(1):57-62.

9. Armagan O, Tascioglu F, Oner C. Eletromyographic biofeedback in the treatment of the hemiplegic hand: a placebo-controlled study. Am J Phys Med Rehabil. 2003;82(11):856-61.

10. Goulart F, Vasconcelos KSS, Souza MRV, Pontes PB. A utilização do biofeedback no tratamento fisioterápico da paralisia facial periférica. Acta Fisiátrica. 2002;9(3):134-40.

11. Calomeni MR, Rocha JAMS, Silva APR, Ribeiro LHB, Marques L, Siza MAF, et al. Brain stimulation used as 
biofeedback training for recovery of motor functions deteriorated by stroke. Arq Neuropsiquiatr. 2013;71(3):159-64.

12. Domingos NAM, Miyazaki MCOS. Emprego do biofeedback no tratamento de doenças crônicas. Arq Ciênc Saúde. 2017;24(1):15-22.

13. Terra NL, Schwanke CHA, Crippa A. O desafio da gerontologia biomédica. $1^{\mathrm{a}}$ ed. Porto Alegre: Edipucrs; 2016. 150 p.

14. Carvalho AC, Barbatto LM, Bofi TC, Silva FA. Estudo da mobilidade funcional de hemiparéticos crônicos tratados com fisioterapia no formato de circuito de treinamento. Revista Adapta. 2015;11(1):19-24.

15. Boni M, Welter MP. Neurociência cognitiva e plasticidade neural: um caminho e ser descoberto. Revista Saberes e Sabores Educacionais. 2016;(3):139-49.

16. Baccaro VM, Kanashiro MS, Batista BP, Beas ARV, Souza CM, Moraes AL. Uso do biofeedback na hidroterapia em pacientes com acidente vascular encefálico. Saúde Rev. 2016;16(42):47-56.

17. Januário PO. Estudo clínico randomizado no tratamento da paralisia facial periférica [dissertação]. São José dos Campos: Universidade do Vale do Paraíba; 2011.

18. Silva JDA, Padovani RC, Viana MB. O emprego do biofeedback como estratégia de manejo do estresse e da ansiedade em atletas: um ensaio clínico. Rev Bras de Ter Comp Cogn. 2016;18(3):17-29.

19. Fernandez-Cuadros ME, Diez-Ramos MF, AlbaladejoFlorin MJ, Perez-Moro OS. Manometric biofeedback effectiveness on urinary incontinence and quality of life: a non-randomized control trial. Middle East J Rehabil Health Stud. 2017;4(2):e46201.

20. Martins M, Palmieri V, Oliveira C. Influência da eletroestimulação parassacral e do biofeedback manométrico na incontinência urinária por hiperatividade do detrusor como sequela de mielite transversa aguda. Fisioterapia Brasil. 2015;16(2):119-23.

21. Byl N, Zhang W, Coo S, Tomizuka M. Clinical impact of gait training enhanced with visual kinematic biofeedback: patients with Parkinson's disease and patients stable post stroke. Neuropsychologia. 2015;79:332-43.

22. Loterio FA. Análise de ativação do padrão muscular de indivíduos hemiparéticos pós-AVC em marcha assistida em andador robótico [dissertação]. Vitória: Universidade Federal do Espírito Santo; 2015.

23. Silva ESM. Correlação do nível de comprometimento da hemiparesia com o uso do membro superior parético [dissertação]. São Carlos: Universidade Federal de São Carlos; 2016.

24. Mesquita RL, Carbone ESM. Tratamento fisioterapêutico nas disfunções sexuais em mulheres após tratamento de câncer ginecológico e de câncer de mama: uma revisão de literatura. Rev Fisioter S Fun. 2015;4(2):32-40.

25. Melo LP, Bezerra VT, Costa VS, Souza FHM, Silveira JCC. Efeitos da terapia espelho na reabilitação do membro superior pós-acidente vascular cerebral. Saúde (St Maria). 2015;41(1):157-64.

\section{Como citar este artigo:}

Pimentel TA, Silva BCP, Silva CKV, Souza JO, Januário PO, Cruz AT. Influência do Biofeedback em pacientes com sequela de acidente vascular cerebral. Rev. Aten. Saúde. 2017;15(53):14-19. 